\title{
研究通迅
}

\section{格的半同态的若干结果 $*$}

本文讨论了格的交同态与并同态之间的 联系,给出了格的交 (并) 同态是并 (交) 同态 (从而是同态) 的充要条件. 作为格的同态 基本定理的推广, 建立了格的半同态基本定 理.

定义 1 设 $f$ 为格 $L$ 到格 $\bar{L}$ 的映射, 若 $\forall a, b \in L, \exists a_{1} \in f^{-1}(f(a)), b_{1} \in f^{-1}(f(b))$ 使

$$
\begin{aligned}
& f\left(a_{1} \wedge b_{1}\right)=f\left(a_{1}\right) \wedge f\left(b_{1}\right), \\
& \left(f\left(a_{1} \vee b_{1}\right)=f\left(a_{1}\right) \vee f\left(b_{1}\right)\right),
\end{aligned}
$$

则称 $f$ 为交 (并) 次同态. 若 $f$ 既为交次同态 又为并次同态, 则称 $f$ 为次同态.

定理 1 格 $L$ 到格 $\bar{L}$ 的交 (并) 同态 $f$ 是 并 (交) 次同态当且仅当 $f(L)$ 是 $\bar{L}$ 的子格.

定义 2 格 $L$ 到格 $\bar{L}$ 的映射 $f$ 称为是射 影的, 如果 $\forall a, b \in L$

$$
f(a \vee b)=f(a) \Leftrightarrow f(a \wedge b)=f(b) .
$$

定理 2 设 $f$ 为格 $L$ 到格 $\bar{L}$ 的交 (并) 同 态, 则 $f$ 是并 (交) 同态 (因而是同态) 的充要 条件是 $f(L)$ 是 $\bar{L}$ 的子格且 $f$ 是射影的.

定义 3 设 $\theta$ 是格 $L$ 的交(并) 合同, $L / \theta$
是 $L$ 的模 $\theta$ 的交 (并) 商半格. 若 $\forall[a],[b] \epsilon$ $L / \theta, \exists a_{0} \in[a], b_{0} \in[b]$, 使 $\forall a^{\prime} \in[a], b^{\prime} \in[b]$ 有

$$
\begin{gathered}
{\left[a_{0} \vee b_{0}\right] \wedge\left[a^{\prime} \vee b^{\prime}\right]=\left[a_{0} \vee b_{0}\right],} \\
\left(\left[a_{0} \wedge b_{0}\right] \vee\left[a^{\prime} \wedge b^{\prime}\right]=\left[a_{0} \wedge b_{0}\right]\right) .
\end{gathered}
$$

规定

$[a] \bigvee[b]=\bigwedge_{\substack{a^{\prime} \in[a] \\ b^{\prime} \in[b]}}\left[a^{\prime} \bigvee b^{\prime}\right]\left([a] \wedge[b]=\bigvee_{\substack{a^{\prime} \in[a] \\ b^{\prime} \in[b]}}\left[a^{\prime} \wedge b\right]\right)$,

则 $L / \theta$ 形成的格称为 $L$ 的模 $\theta$ 的交 (并) 半 商格.

定理 3(格的半同态基本定理) 设 $f$ 为 格 $L$ 到格 $\bar{L}$ 的交(并)同态, 则

(1) $L$ 作为半格有: $f(L)$ 是 $\bar{L}$ 的交(并)子 半格, 并且交 (并) 商半格 $L / \theta_{f} \cong f(L)$;

(2) 若 $f(L)$ 是 $\bar{L}$ 的子格, $L$ 作为格其交 (并)半商格 $L / \theta_{f} \cong f(L)$.

\section{闵佑林}

( 江西教育学院数学与计算机科学系, 南昌 330029)

*江西省自然科学基金资助项目

\section{碳化物团球化与价电子结构}

白口铸铁的基体因被网状碳化物割裂而 韧性较低. 为了提高白口铸铁的韧性, 扩大 其应用范围, 杨相寿等人曾对白口铸铁进行 变质处理, 试图使碳化物断网或团球化 ${ }^{[1]}$. 本课题以低铬白口铸铁为研究对象, 系统地 考察了铸态、正火、淬火 + 回火 3 种状态下不 同加人量的 C, Cr, Mn, Mo V, W, Ti, B, P, Sn, $\mathrm{Sb}, \mathrm{Cu}, \mathrm{Al}, \mathrm{Nb}, \mathrm{Si}, \mathrm{N}$ 等常用合金元素对碳 化物形态及分布的影响, 并且测试了上述 3
种状态下各种成分时铸铁的冲击韧性及洛氏 硬度。结果简述如下:

(1)上述 16 种合金元素加人量的改变对 3 种状态下碳化物团球化的影响各不相同, 其 中大多数合金元素都可以促进碳化物的断网和 成球, 尤以 $\mathrm{V}$ 的作用最为显著. 当 $\mathrm{V}$ 含量 $\geqslant 4 \%$ 时碳化物的断网和孤立极为明显; 当 V含量 $=8 \%$ 时绝大部分碳化物均呈圆球状,仅有少量孤 立块状的碳化物存在. 
(2) 热处理可以促使白口铸铁中的碳化物 断网、成块, 甚至团球化, 从而促使铸铁的韧 性得到提高. 加热温度的影响大于保温时间 的影响. 这是因为在碳化物断网成球过程 中, 需要组元 (碳或合金元素) 原子的扩散. 而根据 Fick 第一扩散定律 ${ }^{[2]}$ :

$$
\varphi=-D_{0} \cdot \exp \left(-\frac{Q}{k T}\right) \cdot \frac{\mathrm{d} c}{\mathrm{~d} x} \cdot t,
$$

式中 $\varphi$ 为在时间 $t$ 内通过单位截面的组元通 量; $D_{0}$ 为扩散常数; $Q$ 为扩散的摩尔激活 能; $R$ 为气体常数; $T$ 为绝对温度; $\frac{\mathrm{d} c}{\mathrm{~d} x}$ 为组 元沿扩散方向 $x$ 的浓度梯度; $t$ 为扩散时间.

因为碳原子在 $\gamma-\mathrm{Fe}$ 中扩散时 $Q=140 \times$ $10^{3} \mathrm{~J} / \mathrm{mol}, R=8.314 \mathrm{~J} / \mathrm{mol} \cdot \mathrm{K}^{-1}, T \leqslant 1050^{\circ} \mathrm{C}=$ $1323.2 \mathrm{~K}$, 代人 $\frac{\partial^{2} \varphi}{\partial T^{2}}$ 和 $\frac{\partial^{2} \varphi}{\partial t^{2}}$, 可知: $\frac{\partial^{2} \varphi}{\partial T^{2}}>0$, $\frac{\partial^{2} \varphi}{\partial t^{2}}=0$, 亦即 $\frac{\partial^{2} \varphi}{\partial T^{2}}>\frac{\partial^{2} \varphi}{\partial t^{2}}$, 所以碳通量
对温度比对时间更为敏感.

为了探讨碳化物的团球化规律及机理, 根据 “经验电子理论” ${ }^{[3,4]}$ 对前述各种白口铸 铁中可能存在的碳化物的价电子结构进行了 分析计算, 结果将陆续发表.

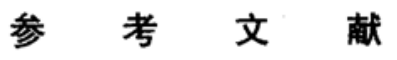

1 杨相寿, 染明汉, 黄重伯. 关于白口铸铁碳化物在铸态 下孤立化与团球化的初步试验. 球铁, 1978, (4): $16 \sim 21$

2 宋维锡主编. 金属学. 北京: 冶金工业出版社, 1980 . $177 \sim 195$

3 余瑞璜. 固体与分子经验电子理论. 科学通报, 1978 , 23(4): $217 \sim 224$

4 余瑞璜. 固体与分子经验电子理论一一等效价电子 假定. 科学通报, 1981, 26(4): 206 209

叶以富 尚玉侠范同祥翟慎秋 (山东工业大学材料工程系, 济南 250014)

\section{锑与硒的负离子质谱}

迄今同位素质谱学对负离子的质谱研究 很少.

锑的电子亲合能较高, 有实现为热电离 负离子的可能, 但也并非十分容易. 作者通 过降低灯丝表面功函数及引人一定正离子到 灯丝表面,在 $9 \mathrm{~mm} \times 0.7 \mathrm{~mm} \times 0.04 \mathrm{mmRe}$ 敷涂

灯丝上, 在约 $4 \mathrm{~A}$ 灯丝电流下, 得到分析化 合物 $\mathrm{Sb}_{2} \mathrm{O}_{3}$ 的 $\mathrm{Sb}^{-}, \mathrm{SbO}^{-}$负离子质谱. 测得 天然同位素丰度比值 $\mathrm{Sb}_{121 / 123}=1.338 \pm 0.007$. 由于负离子热电离选择性高, 极少有杂质本 底峰出现, 谱图简明, 这种优越性在高丰度 测量中表现尤为明显.

取 $\mathrm{NaSeO}_{4} \cdot \mathrm{H}_{2} \mathrm{O}$ 为分析化合物, 选择硅 胶作底物, 敷涂于钽靶上, 在氩的快原子束
轰击下, 获得 $\mathrm{Se}^{-}, \mathrm{SeO}^{-}, \mathrm{SeO}_{2}^{-}$负离子质谱. 在相同条件下还作了无底物净靶实验. 结果 表明, 靶上有无底物硅胶, 被分析物质的电 离效率及其质谱特征均无明显差别. 因此, 如采用硅胶为薄层色谱吸附剂, 以快原子轰 击为扫描电离手段, 这将是一种可取的束靶 组合形式 ${ }^{[1]}$, 它可实现薄层色谱 - 质谱的直 接分析, 若再引人同位素稀释原理, 它对那 些必须进行样品前处理并且要作大量重复测 量的无机定量分析是有意义的.

致谢 硒的实验是在黄庆文研制的 STIMS 型离子质谱计上完成的, 得到了他的支 持与帮助, 在此深表谢意. 